\title{
Clozapine- induced recurrent and transient parotid gland swelling
}

Clozapine is a novel dibenzodiazepine derivative, atypical antipsychotic which exhibits antagonistic activity on 5HT2, alpha 1, alpha 2, $\mathrm{H} 1$ and M1, M2, M3 and M5 receptors; agonist activity at the M4 muscarinic receptor and modest antagonistic effect on D2-like receptors. ${ }^{1}$ Clozapine is known to be superior to other antipsychotics and is effective in $30-50 \%$ of patients diagnosed with treatmentresistant schizophrenia. ${ }^{2}$ However, its use is restricted mainly due to its side effect of agranulocytosis. Hypersalivation is another distressing and common side effect of clozapine which occurs in about $23 \%$ of patients. ${ }^{3}$ Hypersalivation, a poorly understood paradoxical side effect of clozapine, may sometimes cause salivary gland swelling. Rarely, the anticholinergic effect of clozapine may result in the thickening and stasis of saliva, precipitation of calcium salts, and calculus formation in the salivary duct leading to swelling in salivary glands. ${ }^{4}$ In this report, a rare case of clozapine induced parotid gland swelling is described. The aim of bringing this rare but distressing side effect of clozapine to the attention of clinicians is to discuss its pathogenesis. Informed consent to publish this case, on the basis anonymity, was obtained from the patient.

Ms X is a 35 year old female, diagnosed with paranoid schizophrenia, who has received various psychotropic medications. Despite good compliance with the treatment, she suffered from frequent relapses that required recurrent hospital admissions. Two years ago, a diagnosis of treatment-resistant schizophrenia was made and clozapine was commenced. However, she discontinued clozapine treatment due to the development of a parotid gland swelling on the right side, which occurred within a couple of weeks of its commencement. The swelling disappeared within a week after drug discontinuation. She then had trials of other antipsychotics, such as quetiapine,

haloperidol and zuclopenthixol depot, which all resulted in only partial improvement.

During her most recent admission to the hospital, clozapine was again considered due to the recurrence of serious psychotic symptoms and only partial success with other antipsychotic medications. Prominent among her psychotic symptoms was a somatic delusion wherein she believed that there were "bugs" under her skin, and she caused multiple burns to her arms, legs and abdomen with a cigarette lighter in an attempt to burn these "bugs". Her haematological and biochemical investigations and ECG prior to the initiation of clozapine were unremarkable. She

Correspondence

Dr A Vohra

email: akvohra1950@yahoo.com was started on clozapine as per the Clozaril Patient Monitoring Service (CPMS) protocol.

After two weeks of clozapine at the dose of $175 \mathrm{mg}$ per day, she noticed a swelling on her right temporomandibular area in the absence of hyper salivation. There was no change in white blood cell count and she was afebrile. Plasma amylase and lipase levels were not carried out. An otolaryngologist was consulted; and a diagnosis of clozapine induced parotid swelling was suggested. A sialogram was advised, but she refused to consent for this investigation. She felt intimidated, found the swelling to be quite distressing and stigmatising, and declined to continue with clozapine treatment thereafter. Conequently, clozapine was discontinued, and the swelling disappeared after 5 days. She was recommenced on haloperidol, continued lithium carbonate and was discharged to the community mental health team.

The parotid gland swelling in this case was unilateral, recurrent and occurred with the commencement of clozapine, in the absence of any infection or hyper salivation. Additionally, it disappeared following the discontinuation of clozapine, indicating the antipsychotic to be the probable cause. It is further supported by the fact that the swelling had also appeared in the past when Ms X was commenced on clozapine and disappeared when the clozapine was discontinued on the previous occasion. Ms X scored 9 on the adverse drug reaction probability scale designed by Naranjo et al signifying clozapine to be the definite cause for the parotid gland swelling. ${ }^{7}$

The common causes of parotid gland swelling are benign or malignant neoplasms, bacterial infections, viral infections, Sjogren's syndrome, chronic granulomatous disease, clozapine and various other medications and chronic sialadenitis. Sialadenitis may be caused by hypersalivation that can lead to glandular swelling. ${ }^{4,5}$ An immunologically-mediated mechanism has also been proposed for the clozapine-induced parotitis. It is suggested that clozapine-sensitized mononuclear cells are responsible for the development of sialadenitis. ${ }^{6}$

Various mechanisms have been proposed as an explanation for glandular swelling associated with the use of clozapine. The possible pathogenesis of parotid gland swelling in this reported case seems to be similar to that proposed by Vasile and Steingard, who suggested that the anticholinergic effect of clozapine may cause thickening and stasis of saliva with precipitation of calcium salts. This may result in calculus formation and obstruction in the duct leading to parotid gland swelling. ${ }^{4}$

Salivary gland swelling is a rare adverse effect of clozapine which tends to occur early upon the commencement of clozapine therapy. Although it is usually transient, it is distressing and can lead to discontinuation of 
treatment. Various mechanisms have been proposed as possible explanations for the glandular swelling. However, further research is required to understand more

comprehensively the underlying aetiological mechanisms.

A Vohra

Wyre and Fylde CCaTT, Mountcroft, Albert Street, Fleetwood, Lancashire, United Kingdom

\section{References}

1. Buckley FP, Waddington L J. Schizophrenia and Mood Disorders: the new drug therapies in clinical practice (1.st ed). London: Amoid Publishers, 2003

2. Zom SH, Jones SB, Ward KM, Liston DR. Clozapine is a potent and selective muscarinic M4 receptor agonist. Eur J Pharmacol 1994 269: R1-R2

3. Baldessarini RJ, Frankenburg FR. Clozapine: a novel antipsychotic agent. N Engl J Med 1991; 324: 746-754.

4. Vasile JS, Steingard S. Clozapine and the development of salivary gland swelling: a case study. J Clin Psychaitry 1995; 56(11): 511 513.

5. Robinson D, Fenn H, Yesavage J. Possible association of parotitis with clozapine (letter). Am J Psychiatry 1995; 152: 297-298.

6. Brodkin ES, Pelton GH, Price LH. Treatment of clozapine-induced parotid gland swelling. Am J Psychiatry 1996; 153(3): 445.

7. Naranjo CA, Busto U, Sellers EM, et al. A method for estimating the probability of adverse drug reactions. Clin Pharmacol Ther 1981; $30: 239-245$ 